\title{
El aumento de la diferencia de presión arterial sistólica entre brazos se asocia con menor sobrevida
}

\author{
Higher differences in systolic blood pressure between arms are associated with lower survival
}

\section{Objetivo}

Determinar si la diferencia de presión arterial (PA) sistólica (PAS) entre ambos brazos predice una menor sobrevida libre de eventos a diez años.

\section{Diseño, lugar y pacientes}

Estudio de cohorte, realizado en una institución rural de atención general en Devon, Reino Unido, que incluyó una población de pacientes hipertensos bajo tratamiento.

\section{Evaluación de factores pronósticos}

Un único observador no cegado midió la PA en ambos brazos de manera secuencial con un esfigmomanómetro de mercurio, en tres visitas consecutivas, promediándose los registros para obtener un único valor de PA para cada brazo. Se registraron las características demográficas, factores de riesgo cardiovascular y antecedentes de los pacientes.

\section{Medición de resultados principales}

Se recolectó en forma prospectiva información acerca de los eventos de interés: muerte de causa cardiovascular o por cualquier causa (determinada por certificado de defunción) y eventos cardiovasculares (infarto de miocardio o angina de reciente diagnóstico) o cerebrovasculares, diagnosticados en un centro de segundo nivel.

\section{Resultados principales}

Se obtuvieron datos completos de 230 pacientes, 47\% varones, con un promedio de edad de 68,1 años. El $24 \%$ de los

\section{Comentario}

Es importante remarcar que este trabajo es en realidad una prolongación del seguimiento de una cohorte ya publicada por los autores en $2007^{1}$, con resultados similares a los obtenidos en este estudio, y que se trata de pacientes hipertensos ancianos y no de población general.

Como limitaciones, deben remarcarse: 1) el escaso número de pacientes analizados (230), teniendo en cuenta la alta prevalencia de la patología en cuestión; a pesar de lo cual se han encontrado resultados similares en estudios de mayores dimensiones ${ }^{2}$; 2) que no hubo aleatorización del brazo en el que se mediría primero la $\mathrm{PA} ; 3$ ) que no se evaluaron los cambios en el tratamiento antihipertensivo durante el seguimiento y su potencial impacto en los puntos finales del estudio; y 4) que las mediciones de PA en ambos brazos fueron secuenciales y no simultáneas, siendo este último el método recomendado para la investigación epidemiológica ${ }^{3}$. Sin embargo, esta última limitación podría, a mi juicio, entenderse como una fortaleza del estudio, ya que utilizar una técnica de mediciones secuencial es mucho más extrapolable a la vida real y en un sub-análisis de esta misma cohorte se compararon ambas técnicas de pacientes tenían una diferencia de PAS entre ambos brazos de al menos $10 \mathrm{mmHg}$ y el $9 \%$, de al menos $15 \mathrm{mmHg}$. Tras una mediana de seguimiento de 9,8 años, un total de 100 pacientes (44\%) presentaron alguno de los eventos de interés. Las diferencias de PAS entre ambos brazos se asociaron con un aumento en el riesgo de mortalidad por cualquier causa luego de ajustar por el score de Framingham, la PA media, la presencia de diabetes y la pre-existencia de enfermedad vascular (Tabla 1)

Tabla 1. Riesgo ajustado de eventos según el punto de corte de diferencia de PAS

\begin{tabular}{l|c|c}
\multirow{2}{*}{ Evento } & \multicolumn{2}{|c}{ HR ajustado (IC95\%) para diferencias de PAS: } \\
\cline { 2 - 3 } & $\geq \mathbf{1 0} \mathbf{~} \mathbf{m H g}$ & $\geq \mathbf{1 5} \mathbf{~ m m H g}$ \\
\hline Mortalidad global & 3,6 (IC95\% 2 a 6,5) & 3,1 (IC95\% 1,6 a 6) \\
\hline Muerte cardiovascular & 4,2 (IC95\% 1,7 a 10,3) & 2,7 (IC95\% 1,0 a 7,7) \\
\hline Muerte o evento no fatal & 2,8 (IC95\% 1,8 a 4,3) & 2,8 (IC95\% 1,6 a 4,9) \\
\hline
\end{tabular}

HR: Hazard Ratio; PAS: presión arterial sistólica

\section{Conclusiones}

La diferencia de PAS entre ambos brazos se asocia con un aumento del riesgo de eventos cardiovasculares y mortalidad en hipertensos.

Fuente de financiamiento: Scientific Foundation Board of the Royal College of General Practitioners, South West GP Trust y National Institute for Health Research.

medición, obteniendo prevalencias similares en cuanto a diferencia de presión entre ambos brazos ${ }^{4}$.

Por último, es importante destacar que la medición de PA en ambos brazos como práctica rutinaria permite no sólo detectar pacientes con mayor riesgo de eventos debido a diferencias significativas de PA, sino también detectar el brazo de mayor PA del paciente, ya que se ha establecido que medir la PA en un solo brazo puede subestimar el diagnóstico o el grado de control de la hipertensión ${ }^{5}$. El brazo de mayor PA debería ser el elegido para basar las decisiones diagnósticas y terapéuticas, lo cual en la práctica puede requerir, por ejemplo, que al solicitar un estudio de medición ambulatoria de la PA, deba hacerse en este brazo y no en el no dominante, como es la práctica generalizada ${ }^{6}$.

\section{Conclusiones del comentador}

La medición de la PA en ambos brazos tiene implicancias en el ámbito diagnóstico, terapéutico y pronóstico, y debería ser rutinaria en la práctica clínica.

Jessica Barochiner [ Sección Hipertensión Arterial. Servicio de Clínica Médica del Hospital Italiano de Buenos Aires ] jessica.barochiner@ hospitalitaliano.org.ar

Barochiner J. El aumento de la diferencia de presión arterial sistólica entre brazos se asocia con menor sobrevida. Evid Act Práct Ambul Oct-Dic 2012;15(4):130. Comentado de: Clark CE, Taylor RS, Shore AC, y col. The difference in blood pressure readings between arms and survival: primary care cohort study. BMJ. 2012;344:e1327. PMID: 22433975.

\section{Referencias}

1. Clark CE, Powell RJ, Campbell JL. The interarm blood pressure difference as predictor of cardiovascular events in patients with hypertension in primary care: cohort study. J Hum Hypertens. 2007;21:633-6.

2. Agarwal R, Bunaye Z, Bekele D. Prognostic significance of between-arm blood pressure differences. Hypertension. 2008;51:657-662.

3. Lohmann FW, Eckert S, Verberk WJ. Interarm differences in blood pressure should be determined by measuring both arms simultaneously with an automatic oscillometric device. Blood Press Monit. 2011;16(1):37-42.

4. Clark CE, Campbell JL, Powell RJ y col. The inter-arm blood pressure difference and peripheral vascular disease: cross sectional study. Fam Pract. 2007;24:420-6.

5. Karagiannis A, Tziomalos K, Krikis $\mathrm{N}$ y col. The unilateral measurement of blood pressure may mask the diagnosis or delay the effective treatment of hypertension. Angiology. 2005;56(5):565-9.

6. O'Shea JC, Murphy MB. Ambulatory blood pressure monitoring: which arm? J Hum Hypertens. 2000;14(4):227-30. 